\title{
The Role of Lymph Node Metastasis in the Systemic Dissemination of Breast Cancer
}

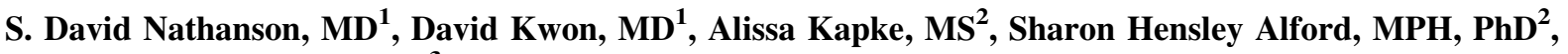 \\ and Dhananjay Chitale, $\mathrm{MD}^{3}$
}

${ }^{1}$ Department of Surgery, Henry Ford Health System, Detroit, MI; ${ }^{2}$ Department of Biostatistics and Research Epidemiology, Henry Ford Health System, Detroit, MI; ${ }^{3}$ Department of Pathology, Henry Ford Health System, Detroit, MI

\begin{abstract}
Background. Lymphatic invasion is necessary for regional lymph node (RLN) metastasis in breast cancer (BC), while systemic metastasis requires blood vessel (BV) invasion. The site of BV invasion could be at the primary $\mathrm{BC}$ site or through lymphovascular anastomoses. The vague pathologic term "lymphovascular invasion" (LVI) encourages the belief that peri/intratumoral BV invasion may be common. We investigated the relative contribution of RLN metastasis to systemic metastasis by studying the relationship among LVI and RLN and/or systemic metastasis in a population-based cohort of breast cancer patients. Methods. Fisher's exact test was done to assess global associations among LVI and RLN and/or systemic metastasis in a prospective database of breast cancer patients undergoing RLN biopsy. Logistic regression was used to determine multivariable contributions of LVI to metastasis when controlling for available demographic, radiologic, and pathologic variables.

Results. Of 1668 patients evaluated $25.4 \%$ were RLN positive and $10.4 \%$ had LVI. RLN metastasis was predicted by tumor size $(P<.0001)$, HER-2/neu overexpression $(P=.0022)$ and the interaction between LVI positive and HER-2/neu positive tumors $(<.0001)$. Patients with LVI/ HER-2-neu positive were 3 times as likely to have positive RLNs compared with patients LVI/HER-2-neu negative.
\end{abstract}

Presented in part at the Sixth Biennial Sentinel Lymph Node meeting, Sydney, Australia, February 2008, and expanded at the 62nd Annual Cancer Symposium of the SSO in Phoenix, AZ, March 2009.

(C) Indian Association of Surgical Oncology 2011

Published Online: 29 March 2011

This article was originally published in Annals of Surgical Oncology, DOI 10.1245/s10434-009-0659-23.

S. D. Nathanson, MD

e-mail: dnathan1@hfhs.org
Systemic metastasis was significantly $(P=.0013)$ associated with LVI/ RLN positive, but not with LVI positive/ RLN negative patients $(P=.137)$.

Conclusions. LVI predicted RLN metastasis. LVI also significantly predicted systemic metastasis, but only when the RLN was also positive. Since RLN requires lymphatic invasion, these data support the hypothesis that primary breast cancers often invade lymphatics to gain access to the systemic circulation.

The systemic metastasis cascade, a concept theorized, confirmed at autopsy by pathologists, clinically and in animal models, and studied in numerous laboratory experiments, suggests that tumor-induced angiogenesis promotes systemic breast cancer (BC) metastases because of direct peritumoral and/or intratumoral invasion of tumor cells into blood vessel capillaries (BVCs). ${ }^{1-3}$ A possible alternative route to the systemic circulation occurs via lymphatic capillary (LC) invasion, passage through peripheral lymphatic trunks, progressing anatomically through the regional lymph nodes (RLNs), the large lymphatic ducts, and the existing lymphaticovenous anastomoses (LVA) in the neck. ${ }^{4}$ Direct local tumoral or peritumoral blood capillary invasion, allowing tumor cells to bypass the RLNs, supports the current surgical paradigm established by Fisher in BC, which led to the belief that BC is often a systemic disease from its origins and that the proper role for complete axillary lymphadenectomy (ALND) is for staging; the therapeutic value of this procedure still vigorously debated. ${ }^{5}$ Systemic metastases established primarily through LC invasion and metastasis to the RLNs would support the Halstedian hypothesis, with a predominant pattern of orderly lymphatic and lymph node progression; systemic metastasis would follow in some patients. ${ }^{6}$ RLN metastasis treated by ALND and/or 
locoregional radiotherapy may produce long-term survival without systemic metastasis, suggesting a stage when tumor cells are confined to the breast and the RLNs and have not yet metastasized to visceral sites. ${ }^{7}$

Pathologists currently allude to the process of peritumoral and/or intratumoral invasion into capillaries as "lymphovascular invasion" (LVI) or use the synonymous term angiolymphatic invasion, electing to avoid the more direct terminology "lymphatic invasion," or "blood vessel invasion," because it is not easy to assuredly distinguish microscopically between LC and BVC using standard morphological criteria after hematoxylin and eosin staining. LVI is associated with a greater likelihood of RLN and systemic metastases, but it is not clear whether systemic metastases in the presence of LVI are always associated with invasion of LCs with an orderly anatomic progression through lymphatic trunks to the RLNs or whether direct local BVC invasion is the predominant method by which BC cells gain access to the systemic circulation. ${ }^{8}$ Descriptions and diagrams of the anatomic pathways of systemic metastasis in textbooks, scientific articles, and reviews demonstrate direct invasion of blood vessels and suggest that angiogenesis not only produces more blood vessels (BVs) that provide greater blood flow to the tumor, but that there is also a greater likelihood of a larger surface "window" of BV endothelium for tumor cells to invade and metastasize. ${ }^{9}$

Preclinical and clinical studies of RLN metastasis and identification of LVI using specific immunohistochemical stains that distinguish lymphatic endothelial cells (LECs) from blood vessel endothelial cells (BECs) strongly suggest that lymphatic invasion is the preferred method by which tumor cells metastasize to the RLNs, but fail to also conclude that this initial lymphatic invasion is also the predominant method by which tumor cells gain access to the systemic circulation. ${ }^{10-13} \mathrm{We}$ interrogated our breast cancer sentinel lymph node (SLN) database, providing a 14-year follow-up of our patients, to determine whether the relationships among LVI, RLN, and systemic metastases support direct peritumoral and/or intratumoral BVC or LC invasion as the predominant pattern of metastasis.

\section{METHODS}

\section{Patients}

All the patients in this study were diagnosed and managed by a multidisciplinary team of breast cancer physicians and ancillary staff in a vertically integrated academic health system. Invasive breast cancer (BC) was diagnosed by image- or palpation-guided core needle biopsy of clinical or radiologic breast abnormalities in most cases, while a tiny proportion of the patients were diagnosed by excision biopsy of a breast lesion in the operating room. All the patients were referred initially to a breast surgeon and preoperatively or postoperatively to specialists in radiation and medical oncology, and occasionally to a plastic surgeon, either by individually arranged office visits, or through a Multidisciplinary Breast Tumor Clinic manned by specialist physicians, breast cancer nurses, social workers, and American Cancer Society volunteers. ${ }^{14}$ The Breast Cancer Multidisciplinary Tumor Board met weekly to discuss each of these patients, reviewing their clinical presentation, radiologic imaging studies, and pathology. This study captures only those breast cancer patients in whom there was no preoperative clinical, radiologic, or pathologic evidence of axillary lymph node metastasis. ${ }^{15}$ The patients were offered an operative sentinel node biopsy (SLNB) by appropriately trained and credentialed surgeons as part of their breast cancer management. ${ }^{16}$ The first few hundred patients undergoing SLNB also underwent a complete axillary lymphadenectomy. ${ }^{16}$

We present here a retrospective cohort study of 1668 clinically node negative patients undergoing 1548 successful SLNBs from April 1995 through April 2009.

The techniques used in the operating room have been reported previously, documenting SLN biopsy, incorporating preoperative intradermal radiocolloid injection and/ or intraoperative injection of $5 \mathrm{~mL}$ of $1 \%$ isosulfan blue or dilute methylene blue into the breast parenchyma adjacent to the breast cancer and into the subareolar lymphatic plexus in the same "clock" position as the tumor. ${ }^{16}$ In all cases where a SLN was not identified, or the patients in whom both procedures were planned and completed as part of the quality assurance, or where the sentinel node(s) contained tumor metastasis a complete axillary lymph node dissection (ALND) was performed. ${ }^{15,16}$ The primary breast tumor was excised by mastectomy or breast-conserving surgery using standard techniques. The terms lumpectomy, partial mastectomy, quadrantectomy, and wide excision were used interchangeably.

All patients were seen by surgical, medical, and radiation oncologists and standard National Comprehensive Cancer Network guidelines used to advise patients regarding the use of these three major modalities of $\mathrm{BC}$ treatment.

The collection of data and the study was approved by the Institutional Review Board.

\section{Pathologic Evaluation}

All primary tumors and axillary lymph nodes were subjected to expert pathologic evaluation by a team of breast pathologists as previously reported. ${ }^{15,16}$ The data were abstracted from pathology reports. In some cases (see 
below) re-review of the tissue sections were done to obtain more LVI information.

Tissue sections were processed using standard pathologic protocols. ${ }^{16}$ A minimum of three sections were submitted from the primary tumor including adjacent uninvolved breast tissue to assess patterns of invasion, distance to the closest surgical margins, and LVI. Other primary breast cancer characteristics were assigned by consensus to each tumor by a team of pathologists, meeting every day to look at each new case with a multiheaded microscope, and the use of a checklist and reported electronically. The checklist included: pTNM stage, anatomic part(s) of the breast involved, histopathologic type, histologic grade (Nottingham), maximum dimension (centimeters), invasive margins (positive or negative), DCIS margins (positive or negative), extensive intraductal component (yes or no), DCIS size, (when feasible), lymph nodes (number with metastasis/number examined), largest node metastasis (centimeters), extracapsular extension, lymphovascular invasion (present or absent, focal or extensive), dermal lymphatic invasion (yes or no), breast tumor markers (estrogen, progesterone, and HER-2-neu receptors; positive or negative), and distant metastasis (assessable or not, yes or no).

Lymphovascular invasion (LVI) was assessed at the periphery of the tumor and intratumoral regions as a standard pathological evaluation. Attention was given to separate true LVI from retraction artifact, often overcalled as LVI. Special immunostain markers to identify endothelial cells were not performed as part of the routine pathologic evaluation. LVI was scored as positive or negative in tumor sections stained with hematoxylin and eosin. Only those patients with unmistakable clusters of tumor cells in the lumen of an endothelial-lined vascular space within or close to the edge of the BC were scored unequivocally positive. Patients with inflammatory breast cancer and dermal lymphatic emboli were excluded. In a small proportion of cases where either of these conclusions could not be conclusively reached, and the pathologist recorded the finding as "suspicious," we elected to classify the patients as negative for purposes of analysis. LVI information was not available initially in 49 patients, and we found, processed, evaluated, and recorded the microscopic tissue slides in 40 , but there was no tissue available for re-review for the other 9 patients.

Lymph nodes were fixed in $10 \%$ buffered formalin and embedded in paraffin. The SLN was serially sectioned along the short axis into 2-mm slices, and 4-6 sections, 5-micron thick, cut at various depths, and standard hematoxylin and eosin staining on glass slides performed. Cytokeratin immunohistochemistry (IHC) was deliberately avoided except to confirm nanometastases in the node, particularly with primary lobular carcinomas. An axillary node was classified as positive (containing metastatic tumor cells) according to standard pathologic criteria and current American Joint Commission on Cancer guidelines.

\section{Patient Follow-Up}

Our large electronic medical record system captured all patients in a longitudinal study, providing accurate details regarding recurrence, and of any deaths. Only a tiny proportion of patients migrated to other health systems, requiring telephone calls and letters by the institutional tumor registry to obtain updated recurrence information. We also accessed the National Death Index Retrieval Program, US Department of Health and Human Services, searching for patients who may have died and whose information was otherwise incomplete.

\section{Data Retrieval, Collection, and Abstracting}

All patient information was available on an electronic medical record system, updated whenever a clinical event occurred. Demographic, clinical, operative, radiological, and pathological information was carefully abstracted by the trained staff in the Department of Biostatistics and Research Epidemiology (BRE) in consultation with the principal investigator (SDN) and recorded in an Excel database. Regular meetings were conducted to discuss data input. The database was periodically updated and searched for errors that were corrected. The database was used for analysis by a biostatistician (AK) and epidemiologist $(\mathrm{SAH})$ in consultation with the senior author.

\section{Statistical Methods}

Logistic regression models were used for all statistical analyses. ${ }^{17}$ Eleven patients had two SLN biopsies, one on each side. If a patient had surgery on both sides on the same date, the side with the positive SLN or with SLN data collected (if no positive SLN found) was used for purposes of length of follow-up analysis. If a patient had 2 surgeries on different dates, data from the second surgery were used. Patients with pathology of "DCIS only," and with negative SLNs, included in our sentinel node data base, were excluded from the analysis $(N=61)$. A total of 1668 patients were included in the initial analysis.

We first tested each predictor of interest in a univariate GEE model. Individual predictors with $P<.10$ were candidates for multivariable modeling. The backward model selection method was used to determine the final multivariable model. Odds ratios (OR) and 95\% confidence intervals $(95 \%$ CI) were calculated for the univariate models and the multivariable model. An OR of 1.0 indicates no association between the predictor and RLN 
positive. An OR $>1$ indicates a positive association between RLN positive and the predictor variable, whereas an $\mathrm{OR}<1$ indicates a negative relationship between RLN positive and the predictor. Model fit was assessed using the c-statistic.

The association between RLN positive, LVI, tumor size, grade, HER-2-neu, ER, PR, and age with systemic metastasis was assessed. First, the individual association between each parameter and metastasis was tested using chi-square tests. All significant parameters $(P<.05)$ were then tested in a multivariable logistic regression model. All possible interactions were tested at $P<.10$.

In an additional analysis, the association between LVI, systemic metastasis, and RLN metastasis was tested using Fisher's exact test.

Kaplan-Meier curves were constructed to depict graphically the relationships among LVI, RLN positive, and systemic metastasis.

\section{RESULTS}

Figure 1 shows the design of the study. A total of 1668 newly diagnosed and previously untreated invasive BC patients with clinically, radiologically, and pathologically negative ipsilateral axillary lymph nodes were evaluated and offered a SLN biopsy as part of their surgical treatment. The median length of follow-up was 3.62 years (mean $=4.15)$. The range was $0-14$ years.

The results of this study are conclusions based on the 174 of 1659 patients $(10.4 \%)$ in whom a definitive pathology report showed invasion of vessels related to the primary tumor (LVI). All patients with RLN data were included in the initial analysis $(N=1668)$.

Table 1 shows the demographics of the study. There were 424 of 1668 patients $(25.4 \%)$ with RLN metastasis; 34 of 120 patients $(28.3 \%)$ whose SLN was not found and who had a complete axillary dissection had metastases in the axillary nodes. There were 9 patients in whom LVI data could not be obtained, and 8 patients in this group with RLN metastases were not included, leaving 407 patients with complete data included.

The first part of the study evaluated predictors of axillary RLN metastasis. Table 2 presents the results from the univariate logistic regression models. Individual predictors of RLN positive included HER-2-neu $(P=.004)$, tumor size $(P<.0001)$, tumor grade $(P<.0001)$, age $(P=$ $.0099)$, and LVI $(P<.0001)$, and these were considered in the multivariable model.

Multivariate analysis identified tumor size $(P<.0001)$, ER $(P=.0048)$, and an interaction between LVI and HER-2-neu (<.0001) as significant predictors of RLN metastasis (Table 3). Patients with tumor size $1-2 \mathrm{~cm}$ (group 2) were 4.5 times more likely to have RLN positive (aOR: 4.52; 95\% CI: 3.54, 5.78). The odds increased to 8.75 for patients with tumor size $\geq 2 \mathrm{~cm}$ (group 3) (aOR: 8.75; 95\% CI: $6.65,11.5)$ compared with those with tumors $<1 \mathrm{~cm}$. Patients who were ER positive were 1.3 times more likely to have RLN positive. Patients who were LVI positive were 14 times more likely than LVI negative patients to have positive RLNs if they had HER-2-neu overexpression (aOR: 14.0; 95\% CI: 1.36, 12.3).The odds of RLN positive for LVI positive vs LVI negative patients was reduced to 4.7 if a patient was HER-2-neu negative (aOR: 4.72; 95\% CI: 3.67, 6.08). The c-statistic for this model was 0.739 , indicating the model has good predictive ability.

The number of patients with systemic metastasis predicted by univariate analysis is presented in Table 4. The final multivariate model included RLN positive by LVI, tumor grade, and tumor size. Results are presented in Table 5. The overall $P$ value for grade was $P=.002$; for tumor size: $P=.012$; for RLN: $P=.011$; and for LVI:

FIG. 1 Study design

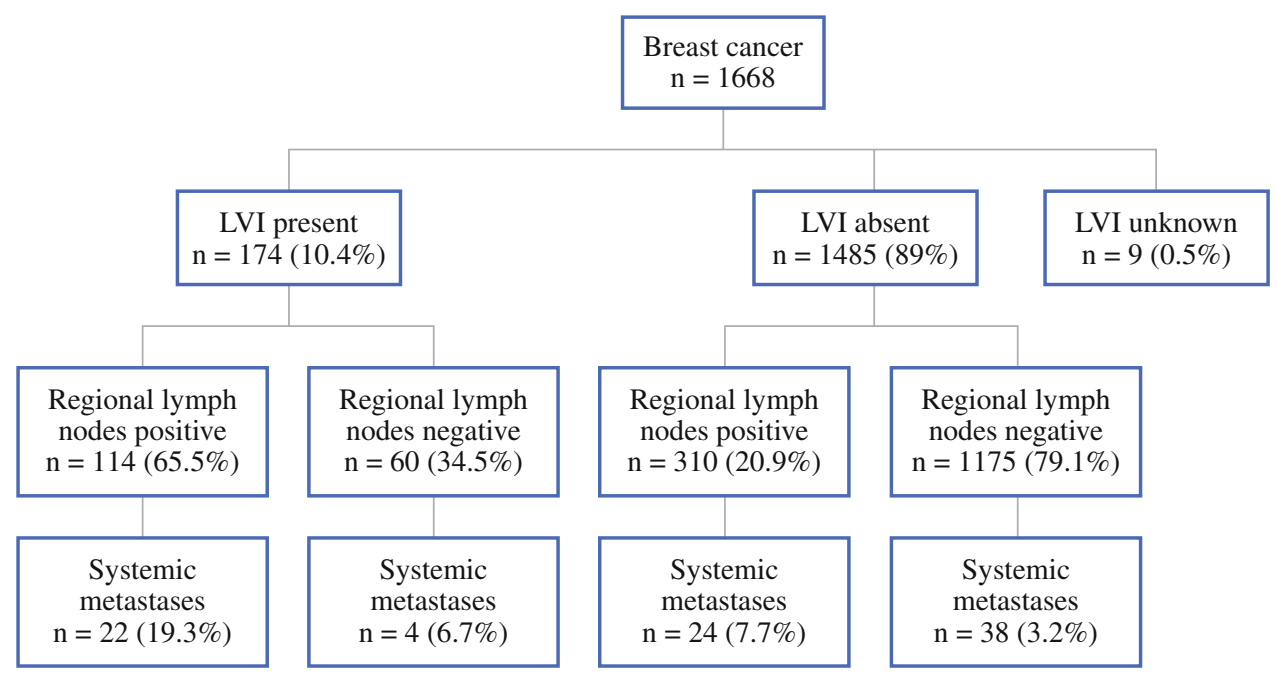


TABLE 1 Demographic information of the patients studied

\begin{tabular}{|c|c|}
\hline Description & $N$ \\
\hline Total cases & 1668 \\
\hline SLN found & 1548 \\
\hline \multicolumn{2}{|c|}{ No. SLNs removed 1.87 (1.49) (mean \pm SD) } \\
\hline RLN tumor positive ${ }^{a}$ & 424 \\
\hline \multicolumn{2}{|c|}{ Tumor size $(\mathrm{cm}) 1.66(1.13)($ mean \pm SD) } \\
\hline$<1$ & 444 \\
\hline $1-2$ & 785 \\
\hline $2-5$ & 411 \\
\hline$>5$ & 21 \\
\hline Unmeasurable & 7 \\
\hline \multicolumn{2}{|l|}{ Tumor grade } \\
\hline 1 & 402 \\
\hline 2 & 759 \\
\hline 3 & 493 \\
\hline Not graded & 14 \\
\hline \multicolumn{2}{|l|}{ Hormone receptors } \\
\hline ER positive & 1301 \\
\hline PR positive & 1207 \\
\hline \multicolumn{2}{|l|}{ HER2-neu } \\
\hline Positive & 205 \\
\hline Negative & 1187 \\
\hline Not performed & 278 \\
\hline \multicolumn{2}{|l|}{ Surgery } \\
\hline Mastectomy & 352 \\
\hline Full axillary dissection & 671 \\
\hline Chemotherapy & 714 \\
\hline Metastases & 89 \\
\hline Lymphovascular invasion & 174 \\
\hline IDCA & 1481 \\
\hline ILCA & 196 \\
\hline
\end{tabular}

${ }^{a}$ Includes all patients with positive axillary nodes, SLN positive, and those in whom the SLN was not found and a complete axillary dissection performed

IDCA invasive ductal carcinoma, ILCA invasive lobular carcinoma, $S L N$ sentinel lymph node, $R L N$ regional lymph nodes

$P=.024$. Results from the model indicated the odds of systemic metastasis were 2.2 times higher among patients who were RLN positive and who had LVI compared with those with negative RLNs and LVI positive. For tumor grade, grade 3 was used as the reference group since only 1 patient with a grade 1 tumor had systemic metastasis. The odds of systemic metastasis among patients with grade 1 tumors were only 0.045 (95\% CI: 0.006, 0.337). Patients with grade 2 tumors were approximately $59 \%$ less likely to have systemic metastasis compared with patients with grade 3 tumors. There was a significant difference in the odds of having systemic metastasis between patients with tumors $>2 \mathrm{~cm}$ versus tumors $<1 \mathrm{~cm}$ (aOR: $2.5 ; 95 \% \mathrm{CI}$ :
TABLE 2 Univariate logistic regression results for RLN positive models

\begin{tabular}{|c|c|c|c|c|}
\hline Variable & $\begin{array}{l}\text { RLN } \\
\text { positive } \\
(N=424)\end{array}$ & $\begin{array}{l}\text { RLN } \\
\text { negative } \\
(N=1244)\end{array}$ & $\begin{array}{l}\text { Odds ratio } \\
(95 \% \mathrm{CI})\end{array}$ & $P$ value \\
\hline \multicolumn{5}{|l|}{ HER-2-neu } \\
\hline Positive & $64(19 \%)$ & $141(13 \%)$ & $1.52(1.14,2.03)$ & .004 \\
\hline Negative & $272(81 \%)$ & $913(87 \%)$ & & \\
\hline \multicolumn{5}{|l|}{ ER } \\
\hline Positive & $326(77 \%)$ & $975(79 \%)$ & $0.92(0.79,1.06)$ & .235 \\
\hline Negative & $95(23 \%)$ & $260(21 \%)$ & & \\
\hline \multicolumn{5}{|l|}{ PR } \\
\hline Positive & $302(72 \%)$ & $905(73 \%)$ & $0.92(0.75,1.13)$ & .408 \\
\hline Negative & $120(28 \%)$ & $330(27 \%)$ & & \\
\hline \multicolumn{5}{|l|}{ Tumor size } \\
\hline$<1$ & $32(8 \%)$ & $412(33 \%)$ & Reference & \\
\hline $1-2$ & $201(48 \%)$ & $584(47 \%)$ & $4.41(2.97,6.54)$ & $<.0001$ \\
\hline$\geq 2$ & $188(45 \%)$ & $244(20 \%)$ & $9.97(6.69,14.86)$ & $<.0001$ \\
\hline \multicolumn{5}{|l|}{ Grade } \\
\hline 1 & $53(13 \%)$ & $349(28 \%)$ & Reference & \\
\hline 2 & $205(49 \%)$ & $554(45 \%)$ & $2.44(1.56,3.80)$ & $<.0001$ \\
\hline 3 & $161(38 \%)$ & $332(27 \%)$ & $3.19(2.46,4.14)$ & $<.0001$ \\
\hline \multicolumn{5}{|l|}{ Age } \\
\hline$<50$ & $102(24 \%)$ & $227(18 \%)$ & $0.71(0.54,0.92)$ & .0099 \\
\hline$\geq 50$ & $322(76 \%)$ & $1014(82 \%)$ & & \\
\hline \multicolumn{5}{|c|}{ Lymphovascular invasion } \\
\hline Positive & $114(27 \%)$ & $60(5 \%)$ & $7.20(6.01,8.63)$ & $<.0001$ \\
\hline Negative & $310(73 \%)$ & $1174(95 \%)$ & & \\
\hline
\end{tabular}

$E R$ estrogen receptor, $P R$ progesterone receptor, $R L N$ regional lymph nodes

TABLE 3 Multivariable model with tumor size, ER, and HER-2-neu by LVI interaction and the relationship to the RLN

\begin{tabular}{|c|c|c|}
\hline Variable & Odds ratio $(95 \% \mathrm{CI})$ & $P$ value \\
\hline \multicolumn{3}{|l|}{ Tumor size } \\
\hline$<1$ & Reference group & \\
\hline $1-2$ & $4.52(3.54,5.78)$ & $<.0001$ \\
\hline$\geq 2$ & $8.75(6.65,11.5)$ & $<.0001$ \\
\hline ER: positive vs. negative & $1.28(1.08,1.53)$ & .0048 \\
\hline \multicolumn{3}{|l|}{ HER-2-neu*Lymphatic invasion } \\
\hline HER2 $^{+}$vs. HER2 ${ }^{-}:$LVI $^{+}$ & $4.09(1.36,12.3)$ & .012 \\
\hline $\mathrm{HER}^{+}{ }^{+}$vs. HER2 ${ }^{-}: \mathrm{LVI}^{-}$ & $1.38(1.03,1.83)$ & .029 \\
\hline $\mathrm{LVI}^{+}$vs. $\mathrm{LVI}^{-}:$HERer ${ }^{+}$ & $14.0(4.63,42.6)$ & $<.0001$ \\
\hline $\mathrm{LVI}^{+}$vs. $\mathrm{LVI}^{-}: \mathrm{HER}^{-}$ & $4.72(3.67,6.08)$ & $<.0001$ \\
\hline
\end{tabular}

$1.00,6.32 ; P=.05)$. Patients with tumor size $1-2 \mathrm{~cm}$ were approximately $53 \%$ less likely to have metastasis compared with patients with tumors $>2 \mathrm{~cm}$ (OR: 0.47; 95\% CI: 0.28 , $0.81 ; P=.007$ ). The c-statistic for this model (Table 5) is 0.796 . 
TABLE 4 Univariate logistic regression results for prediction of systemic metastasis

\begin{tabular}{lccr}
\hline Variable & $\begin{array}{l}\text { No. metastasis } \\
(N=1576)\end{array}$ & $\begin{array}{l}\text { Metastasis } \\
(N=89)\end{array}$ & $\begin{array}{l}P \\
\text { value }\end{array}$ \\
\hline $\begin{array}{l}\text { RLN positive } \\
\text { HER-2-neu } \\
\text { positive }\end{array}$ & $377(24 \%)$ & $46(52 \%)$ & $<.001$ \\
ER positive & $190(14 \%)$ & $15(22 \%)$ & .086 \\
PR positive & $1159(74 \%)$ & $52(59 \%)$ & $<.001$ \\
Grade & & $46(52 \%)$ & $<.001$ \\
$\quad 1$ & $400(26 \%)$ & $2(2 \%)$ & $<.001$ \\
2 & $722(46 \%)$ & $34(38 \%)$ & \\
$\quad 3$ & $440(28 \%)$ & $53(60 \%)$ & \\
Age $\geq 50$ & $1268(81 \%)$ & $66(74 \%)$ & .142 \\
LVI & $147(9 \%)$ & $26(30 \%)$ & $<.001$ \\
Tumor size & & $8(9 \%)$ & $<.001$ \\
$\quad<1$ & $433(28 \%)$ & $29(33 \%)$ & \\
$1-2$ & $748(48 \%)$ & $51(58 \%)$ & \\
$>2$ & $375(24 \%)$ & & \\
\hline
\end{tabular}

TABLE 5 Final logistic regression model for prediction of systemic metastasis

\begin{tabular}{lllll}
\hline Comparison & OR & $\begin{array}{l}95 \% \text { CI } \\
\text { lower bound }\end{array}$ & $\begin{array}{l}\text { 95\% CI } \\
\text { upper bound }\end{array}$ & $P$ value \\
\hline RLN & 1.89 & 1.16 & 3.08 & .011 \\
LVI & 1.87 & 1.08 & 3.22 & .024 \\
Grade 1 vs. 3 & 0.078 & 0.018 & 0.33 & .005 \\
Grade 2 vs. 3 & 0.479 & 0.30 & 0.76 & .002 \\
Tumor group 2 vs. 1 & 1.21 & 0.54 & 2.74 & .645 \\
Tumor group 3 vs. 1 & 2.82 & 1.26 & 6.32 & .012 \\
\hline
\end{tabular}

The association between LVI, RLN positive, and systemic metastasis was tested using Fisher's exact test (Tables 6,7). There was no association between LVI and systemic metastases for RLN negative patients $(P=.137)$. Among RLN positive patients, systemic metastasis was significantly $(P=.0013)$ higher among patients with LVI (19\%) compared with LVI negative patients $(6.8 \%)$. We included 423 RLN positive for this analysis because we had 1 RLN positive patients with missing LVI data.

Figures 2, 3, 4, 5 show Kaplan-Meier constructs of systemic disease and the interaction of LVI and RLN. Figure 2 shows a significant $(P<.0001)$ increase in systemic metastases in patients with both LVI and RLN positive compared with those with neither LVI or RLN metastases. Figure 3 shows insignificant (.246) differences in systemic metastasis for those patients who had negative RLNs, whether or not their tumors showed LVI. Figure 4 shows patients whose tumors exhibited LVI; those with positive RLNs had significantly $(P=.023)$ more systemic
TABLE 6 LVI and systemic metastases for RLN negative patients

\begin{tabular}{lrrrr}
\hline Lymphatic invasion & \multicolumn{2}{l}{ Metastases } & Total & $P$ value \\
\cline { 2 - 3 } & \multicolumn{1}{l}{ No } & \multicolumn{2}{c}{ Yes } & \\
\hline No & $1137(96.8)$ & $38(3.2)$ & 1175 & .137 \\
Yes & $55(93.2)$ & $4(6.8)$ & 59 & \\
\hline
\end{tabular}

TABLE 7 LVI and systemic metastases for RLN positive patients

\begin{tabular}{lrlll}
\hline Lymphatic invasion & \multicolumn{2}{c}{ Metastases } & Total & $P$ value \\
\cline { 2 - 3 } & \multicolumn{1}{l}{ No } & \multicolumn{2}{c}{ Yes } & \\
\hline No & $285(92.2)$ & $24(7.8)$ & 309 & .0013 \\
Yes & $92(80.7)$ & $22(19.3)$ & 114 & \\
\hline
\end{tabular}

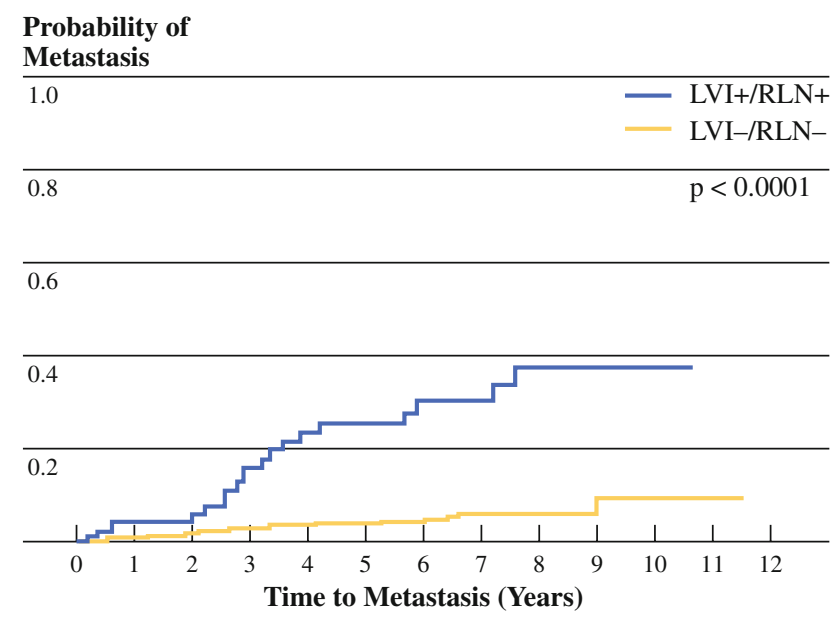

FIG. 2 Kaplan-Meier plots of systemic metastases comparing RLN negative/LVI negative to RLN positive/LVI positive $(P<.0001)$

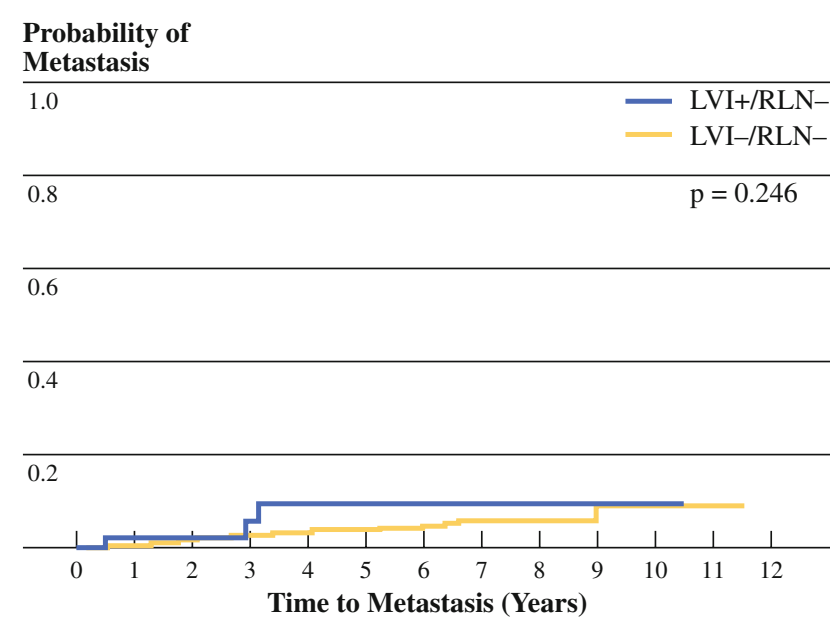

FIG. 3 Kaplan-Meier plots of systemic metastases comparing RLN negative/LVI negative to RLN negative/LVI positive $(P=.246)$ 
Probability of

Metastasis

\begin{tabular}{lr}
\hline 1.0 & - LVI+/RLN+ \\
& LVI+/RLN- \\
\hline 0.8 & $\mathrm{p}=0.023$
\end{tabular}

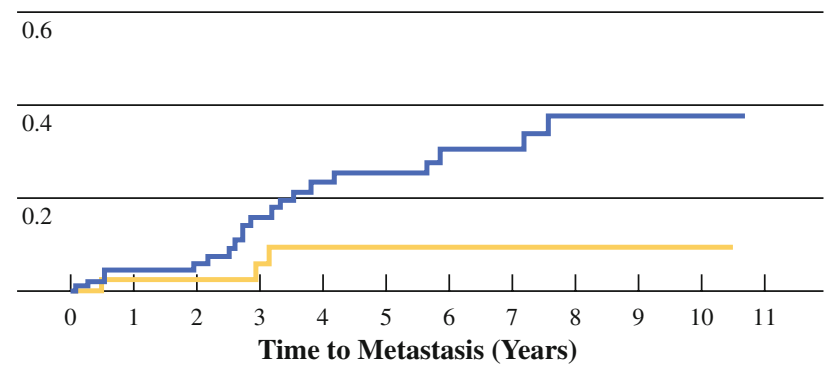

FIG. 4 Kaplan-Meier plots of systemic metastases comparing RLN positive/LVI positive to RLN negative/LVI positive $(P=.023)$

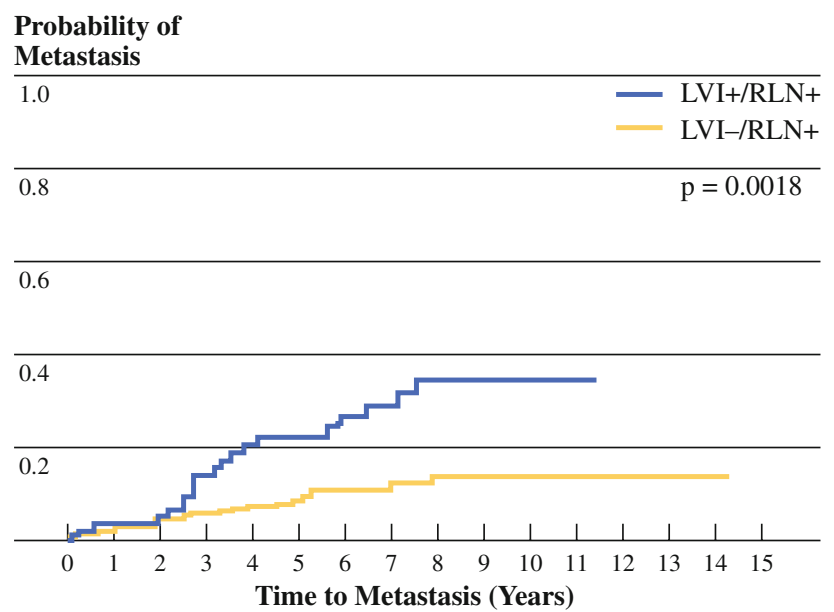

FIG. 5 Kaplan-Meier plots of systemic metastases comparing RLN positive/LVI negative to RLN positive/ LVI positive $(P=.0018)$

metastases compared with those with negative RLNs. Figure 5 compares those patients with RLN metastases in which one group had LVI and the other did not, showing a significantly $(P=.0018)$ higher incidence of systemic metastases in those with both RLN and LVI positive.

\section{DISCUSSION}

Tumor invasion into endothelial-lined vascular spaces, called lymphovascular invasion (LVI) by pathologists, was significantly associated with both regional lymph node (RLN) and systemic metastases in this study of 1668 clinically N0 breast cancer patients offered sentinel lymph node biopsy and/or complete ALND and followed for 114 years (median 3.62 years). Analysis of the data also showed that systemic metastasis in the presence of LVI required metastasis to the RLNs for the association to be true, supporting the likelihood that the invaded vessels were lymphatics rather than blood vessels.

Systemic breast cancer metastasis has been extensively studied preclinically, clinically, and in vitro. ${ }^{1,4,10}$ Extensive work in the metastatic cascade yielded much information about the key steps in the process. ${ }^{1,18}$ Tumor cells invade breast parenchymal tissue, a process requiring proteolysis, adhesion receptors, and directional motility, but little is known about the initial steps whereby the cells interact with tumoral or peritumoral vessels and gain access to the systemic circulation. ${ }^{19,20}$

While tumors can enter the blood directly by intravasating into blood vessel capillaries or indirectly via lymphatics the relative contributions of both pathways as routes of egress from the primary site is still debated. ${ }^{4}$ Direct observation by videomicroscopy in an animal tumor model of preferential tumor cell invasion into peritumoral lymphatics has yet to be demonstrated in human breast cancer. ${ }^{21}$ Highly specific lymphatic endothelial immunohistochemical stains allowed direct pathological observation that BC invaded lymphatics in close to $100 \%$ of cases when LVI was present. ${ }^{13}$ Other clinical and preclinical studies showed a correlation between sentinel node metastasis and lymphangiogenesis, stimulated by VEGF$\mathrm{A}, \mathrm{C}$, and $\mathrm{D}$ in the tumor microenvironment, suggesting a higher likelihood of lymphatic invasion with a larger surface area of lymphatic endothelium. ${ }^{10,22-24}$. Clinical and animal epithelium-derived tumors usually invaded lymphatics and metastasized first to the RLNs. ${ }^{10,24-26}$

Physiologic and pathophysiologic studies provide further indirect evidence that tumor cells are more likely to invade peritumoral lymphatics than blood vessels. Lymphatic capillaries lack interendothelial tight junctions, pericytes, and basement membranes seen in blood vessels. ${ }^{27,28}$ High intratumoral interstitial fluid pressure (IFP), caused by abnormal structure and function of blood and lymphatic vessels in tumors, results in significant fluid convection near the tumor margin, creating peritumoral edema, transport of tumor-derived molecules and cells that generate lymphangiogenesis and angiogenesis, and increased lymphatic flow. ${ }^{21,27-31}$ Interstitial hypertension compresses blood vessels but, because of anchoring filaments that tether LECs to surrounding stromal tissues, and overlapping endothelial cells that open up gaps in the lymphatic capillary wall, lymphatic lumena may remain open despite the increased pressure and mechanically enhance the intravasation of fluid and tumor cells. ${ }^{28,32}$ The low-shear lymphatic system is also less likely to cause mechanical destruction of tumor cells than the high-shear blood circulation. ${ }^{18}$

Some of the earlier studies in the mechanisms involved in lymph node metastasis focused heavily on the physiology and pathophysiology of the RLN. The possible 
"barrier function" of RLNs in cancer was addressed more than 40 years ago, showing a short transit time of cells through nodes and a suggestion that there was a very close association between lymphatics and blood vessels. ${ }^{33}$ It seemed likely that the majority of tumor cells traversing the lymph nodes either entered the efferent lymphatic or the venous system through lymphaticovenous communications. Some studies showed direct lymphaticovenous connection in lymph nodes using air or bacteria, radioactive chromium, and radio-opaque contrast material in patients. ${ }^{34-36}$ Willis, upon careful pathological evaluation of patients, suggested that hematologic dissemination of tumors occurred via lymphatic tributaries to veins in RLNs. ${ }^{37}$ Other studies failed to show such a connection using radiolabeled erythrocytes. ${ }^{38}$ Recent experiments demonstrate tumor cytokine-induced sentinel lymph node angiogenesis and lymphangiogenesis, suggesting an anatomic pathway for tumor cells in the lymph node to migrate from lymphatics through adjacent dilated high-endothelial venules, although this pathway has not yet been documented directly. ${ }^{23,39}$

A direct association between lymphatic and hematogenous metastasis has been noted in many clinical studies. ${ }^{40-42}$ Although it is difficult to conclude the order of events with certainty, the common observation in all these studies points to the likelihood that RLN metastasis occurs before hematogenous metastasis. The orderly progression of common cancers through the lymphatic system to the blood stream has also been described in animal tumor models. ${ }^{10,25}$

Intramammary lymphatic tumor emboli were strongly correlated with RLN and systemic metastasis in breast cancer patients when regular sections were stained with hematoxylin and eosin. ${ }^{8}$ The incidence of blood vessel invasion was reported in $4.7 \%$, while others reported this phenomenon in $71 \%$ of patients. ${ }^{19,43}$ The conclusions from a number of investigators in the 1960s and 1970s clearly demonstrate a strong belief that systemic metastases occurred when intramammary blood vessels were invaded; invasion of intramammary lymphatics was thought to be associated with RLN metastasis but not necessarily with systemic metastases. ${ }^{44,45}$ Modern studies acknowledge the difficulty in distinguishing blood and lymph vessels. The availability of immunohistochemical techniques that specifically stain lymphatics enabled the pathologists in one study to confidently identify vascular invasion to be lymphatic in $96.4 \%$ of cases; they also found a significant correlation with RLN metastasis and lymphatic invasion. ${ }^{13}$ Our study, while it did not pathologically identify LVI as lymphatic, certainly found the same clinical association with presumed lymphatic invasion and both RLN and systemic metastases.

It is reasonable to assume that all the RLN-positive patients had LVI, but in those patients we only found 174 of $423(41 \%)$ with LVI. This probably represents a sampling error; if every micron of the tumor were evaluated LVI would likely be found in a much higher number. Similarly, in those patients whose primary tumors exhibited LVI but failed to show RLN metastasis we could expect that a higher proportion of positive nodes would be found if more than a small fraction of the node were evaluated. Routine histologic examination of axillary lymph nodes in patients with breast cancer significantly underestimates lymph node metastasis. ${ }^{46}$ Some of the RLNs were likely truly negative since intramammary lymphatic tumor emboli likely take time in transit and may not reach the RLNs.

Hematogenous dissemination may occur without identifiable RLN metastasis. Direct blood vessel invasion may occur in some patients in whom no lymphatic invasion occurs. Patients harboring bone marrow micrometastases in the absence of RLN metastases have been reported in $20 \%-40 \%$ of carcinomas. ${ }^{47}$ Experimental and clinical studies in other tumor types have shown mostly apoptotic or dead tumor cells shedding into the venous effluent of tumors. ${ }^{9,48}$ Proof of truly independent RLN and hematogenous metastases requires a genomic approach that has not yet been reported.

The analysis presented in this paper does not include patients with clinically and/or radiologically identified axillary lymph node positive cases diagnosed preoperatively. Patients with more advanced locoregional disease than the cases studied here are more likely to have systemic metastases. ${ }^{5,12,26}$ Because such advanced cases would have progressed through the same stages as the patients presented in this paper, it is unlikely that the addition of those patients would alter our conclusions.

Our analysis does not directly contradict the paradigms currently used for surgical treatment of breast cancer. The continued debate about the therapeutic efficacy of complete axillary lymphadenectomy for sentinel node positive breast cancer can only be resolved by carefully planned and executed randomized prospective clinical studies such as the elegantly designed but incomplete ACOSOG Z0011 study.

In summary, our study clearly supports the Nottingham group who provided direct histologic evidence in human breast cancer that LVI is predominantly ( $>95 \%$ of cases) lymphatic invasion. ${ }^{13}$ Our data showed that LVI is a predictor of both RLN and systemic metastasis. The likelihood of systemic metastasis was extremely small and statistically insignificant when LVI was observed and where no RLN metastasis was found. RLN metastasis was an independent predictor of systemic metastasis and the likelihood of metastasis was higher and statistically significant when both LVI and RLN metastasis occurred together, particularly when the tumor overexpressed HER-2-neu. This 
information could prove valuable as a preoperative predictor of systemic disease in patients with breast cancer who also have RLN metastasis.

ACKNOWLEDGMENT Supported by the Nathanson/Rands Chair in Breast Cancer Research. Patricia Baker did the abstracting for the database.

\section{REFERENCES}

1. Fidler IJ. Critical determinants of metastasis. Semin Cancer Biol. 2002;12:89-96.

2. Folkman J. Role of angiogenesis in tumor growth and metastasis. Semin Oncol. 2002;29:15-8.

3. Carmeliet P, Jain RK. Angiogenesis in cancer and other diseases. Nature. 2000;407:249-57.

4. Nathanson SD. Insights into the mechanisms of lymph node metastasis. Cancer. 2003;98:413-23.

5. Punglia RS, Morrow M, Winer EP, Harris JR. Local therapy and survival in breast cancer. N Engl J Med. 2007;356:2399-405.

6. Halsted W. The results of operations for the cure of carcinoma of the breast. Ann Surg. 1907;46:1-9.

7. Hellman S. Karnovsky Memorial Lecture. Natural history of small breast cancers. J Clin Oncol. 1994;12:2229-34.

8. Nime FA, Rosen PP, Thaler HT, Ashikari R, Urban JA. Prognostic significance of tumor emboli in intramammary lymphatics in patients with mammary carcinoma. Am J Surg Pathol. 1977;1:2530.

9. Liotta LA, Kleinerman J, Saidel GM. Quantitative relationships of intravascular tumor cells, tumor vessels and pulmonary metastases following tumor implantation. Cancer Res. 1974;34:997-1004.

10. Nathanson SD. Preclinical models of regional lymph node tumor metastasis. In: Leong SPL, editor. Cancer metastasis and the lymphovascular system: basis for rational therapy. New York: Springer, 2007. p. 129-56.

11. Reintgen D, Cruse CW, Wells K. The orderly progression of melanoma nodal metastases. Ann Surg. 1994;220:759-67.

12. Foster RS. The biologic and clinical significance of lymphatic metastases in breast cancer. Surg Oncol Clin NAm. 1996;5:79-104.

13. Mohammed RAA, Martin SG, Gill MS, Green AR, Paish EC, Ellis IO. Improved methods of detection of lymphovascular invasion demonstrate that it is the predominant method of vascular invasion in breast cancer and has important clinical consequences. Am J Surg Pathol. 2007;31:1825-33.

14. Gabel M, Nathanson SD, Hilton N. Multidisciplinary breast cancer clinics: do they work? Cancer. 1997;79:2380-4.

15. Nathanson SD, Slater R, Kapke A, Burke M. Preoperative identification of the sentinel lymph node. Ann Surg Oncol. 2007; 14:3102-10.

16. Nathanson SD, Grogan J, DeBruyn D, Kapke A, Karvelis K. Breast cancer sentinel node identification rates: the influence of radiocolloid mapping, case volume and the place of the procedure. Ann Surg Oncol. 2007;14:1629-37.

17. Liang KY, Zeger SL. Longitudinal data analysis using generalized linear models. Biometrika. 1986;73:13-22.

18. Buckhorn M, Jain RK, Munn LL. Active versus passive mechanisms in metastasis: do cancer cells crawl into vessels, or are they pushed? Lancet Oncol. 2007;8:444-8.

19. Fisher E, Gregorio R, Fisher B. The pathology of invasive breast cancer. Cancer. 1975;36:1-85.

20. Wong SY, Hynes RO. Lymphatic or hematogenous dissemination: how does a metastatic tumor cell decide? Cell Cycle. 2006;5:812-7.
21. Hoshida T, Isaka N, Hagendoorn J, di Tomaso E, Chen YL, Pytowski B, et al. Imaging steps of lymphatic metastasis reveals that vascular endothelial growth factor-C increases metastasis by increasing delivery of cancer cells to lymph nodes: therapeutic implications. Cancer Res. 2006;66:8065-75.

22. Nathanson SD, Zarbo RJ, Wachna DL, Spence CA, Andrzejewski TA, Abrams J. Microvessels that predict axillary lymph node metastases in Breast Cancer. Arch Surg. 2000;135:586-94.

23. Hirakawa S, Kodama S, Kunstfield R, Kajiya K, Brown LF, Detmer M. VEGF-A induces tumor and sentinel node lymphangiogenesis and promotes lymphatic metastasis. J Exp Med. 2005;201:1089-99.

24. He Y, Rajantie I, Pajusola K, Jeltsch M, Holopainen T, YlaHerttuala S, et al. Vascular endothelial growth factor receptor 3mediated activation of lymphatic endothelium is crucial for tumor cell entry and spread via lymphatic vessels. Cancer Res. 2005;65:4739-46.

25. Yoshizawa M, Shingaki S, Nakajima T, Saku T. Histopathological study of lymphatic invasion in squamous carcinoma $(\mathrm{O}-$ $1 \mathrm{~N})$ with high potential of lymph node metastasis. Clin Exp Metastasis. 1994;12:347-56.

26. Balch CM, Soong S-J, Gershenwald JE, Thompson JF, Reintgen DS, Cascinelli N, et al. Prognostic factors analysis of 17,600 melanoma patients: validation of the American Joint Committee on Cancer, Melanoma Staging System. J Clin Oncol. 2001;19:3622-34.

27. Alitalo K, Carmeliet P. Molecular mechanisms of lymphangiogenesis in health and disease. Cancer Cell. 2002;1:219-27.

28. Schmid-Schonbein GW. Microlymphatics and lymph flow. Physiol Rev. 1990;70:987-1028.

29. Jain RK. Transport of molecules in the tumor interstitium: a review. Cancer Res. 1987;47:3038-50.

30. Jain RK, Tong RT, Munn LL. Effect of vascular normalization by antiangiogenic therapy on interstitial hypertension, peritumor edema, and lymphatic metastasis: insights from a mathematical model. Cancer Res. 2007;67:2729-35.

31. Avery M, Nathanson SD, Hetzel FW. Lymph flow from murine footpad tumors before and after sublethal hyperthermia. Radiat Res. 1992;132:50-3.

32. Padera TP, Stoll BR, Tooredman JB, Capen D, diTomaso E, Jain RK. Pathology: cancer cells compress intratumour vessels. Nature. 2004;427:695.

33. Fisher B, Fisher E. Barrier function of lymph node to tumor cells and erythrocytes: I. Normal nodes. Cancer. 1967;20:1907-13.

34. Pressman JJ, Simon MB. Experimental evidence of direct communications between lymph nodes and veins. Surg Gynec Obstet. 1961;113:537-41.

35. Borodin YI, Tomchek GV. Functional relationships between blood vessels and lymphatic sinuses normally and during experimental disturbances of blood and lymph circulation. Fed Proc. 1966;25T:778-9.

36. Bron KM, Baum S, Abrams HL. Oil embolism in lymphangiography-incidence, manifestations and mechanisms. Radiology. 1963;80:194-202.

37. Willis RA. Pathology of tumours. 3rd ed. London: Butterworth; 1960. p. 167-72.

38. Sabiston DC, Archer GW, Blalock A. Fate of cells in passage through lymphatic and lymph nodes. Ann Surg. 1963;158:570 78.

39. Qian CN, Berghuis B, Tsarfaty G, Bruch M, Kort EJ, Ditleve J, et al. Preparing the "soil": the primary tumor induces vasculature reorganization in the sentinel lymph node before the arrival of metastatic cancer cells. Cancer Res. 2006;66:10365-76.

40. Cianfrocca M, Goldstein LJ. Prognostic and predictive factors in early-stage breast cancer. Oncologist. 2004;9:606-16. 
41. Bubendorf L, Schopfer A, Wagner U, Sauter G, Moch H, Willi N, et al. Metastatic patterns of prostate cancer. An autopsy study of 1589 patients. Hum Pathol. 200;31:578-82.

42. Yoshida T, Matsumoto T, Saski A, Shibata K, Aramaki M, Kitano S. Outcome of para-aortic node positive pancreatic head and bile duct adenocarcinoma. Am J Surg. 2004;187:736-40.

43. Friedell GH, Betts A, Sommers AC. The prognostic value of blood vessel invasion and lymphocytic infiltrates in breast carcinoma. Cancer. 1965;18:164-6.

44. Ruiz U, Babeu S, Schwartz MS, Soto E, McAuley RA, Friedell GH. Blood vessel invasion and lymph node metastasis. Surgery. 1973;73:185-90.
45. Kister SJ, Sommers SC, Haagensen CD, Cooley E. Re-evaluation of blood vessel invasion as a prognostic factor in carcinoma of the breast. Cancer. 1966;19:1213-6.

46. Chagpar A, Middleton LP, Sahin AA, Meric-Bernstam F, Kuerer HM, Feig BW, et al. Clinical outcome of patients with lymph node-negative breast carcinoma who have sentinel lymph node micrometastases detected by immunohistochemistry. Cancer. 2005;103:1581-6.

47. Pantel K, Cote RJ, Fodstad O. Detection and clinical importance of micrometastatic disease. J Natl Cancer Inst. 1999;91:1113-24.

48. Butler TP, Gullino PM. Quantitation of cell shedding into efferent blood of mammary adenocarcinoma. Cancer Res. 1975;35:512-6. 